\title{
Parent-Child Relations, Conduct Problems and Cigarette Use in Adolescence: Examining the Role of Genetic and Environmental Factors on Patterns of Behavior
}

\author{
Katherine H. Shelton • Gordon T. Harold • \\ Tom A. Fowler · Frances J. Rice • Michael C. Neale • \\ Anita Thapar · Marianne B. M. van den Bree
}

Received: 13 September 2007 / Accepted: 7 November 2007/Published online: 22 November 2007

(C) The Author(s) 2007

\begin{abstract}
This study investigated genetic and environmental influences on the associations between mother-child relationship quality (warmth and hostility) and adolescent conduct problems and cigarette use. Participants included 601 mothers and adolescent twin pairs (aged 12-17 years). Mothers and adolescents provided separate reports of mother-to-child warmth and hostility. A combined measure of mother and adolescent reported conduct problems was used while adolescents provided reports of their cigarette use. Analyses were conducted using bivariate genetic analyses of correlated factors models and regression analyses of monozygotic twin differences. Genetic influences were found for most ratings of the parent-child relationship, with evidence of gender and/or rater-specificity for some measures. The relationship between mother-child hostility with adolescent conduct problems and cigarette use was influenced by genetic and environmental effects. Evidence was found for shared environment effects on the relationship between mother-child warmth and conduct problems. Examining monozygotic twin differences provided further support for non-shared environmental influence on the relationship between mothers' expressions of hostility and low warmth and adolescent adjustment. Findings are
\end{abstract}

K. H. Shelton $(\varangle) \cdot$ G. T. Harold

School of Psychology, Cardiff University, Cardiff CF10 3AT,

UK

e-mail: Sheltonkh1@ cardiff.ac.uk

T. A. Fowler - F. J. Rice - A. Thapar - M. B. M. van den Bree Department of Psychological Medicine, Cardiff University, Heath Park, Cardiff CF14 4XN, UK

M. C. Neale

Department of Psychiatry and Human Genetics, Virginia

Commonwealth University, Richmond, VA, USA discussed in relation to the interplay between genetic and environmental effects underlying links between parentchild relations and adolescent behavior problems.

Keywords Conduct problems - Smoking - Genetic · Environmental mediation · Adolescence .

Parent-child relations

\section{Introduction}

In a recent review of child well-being in OECD countries (Organisation for Economic Co-operation and Development member), the United Kingdom, alongside the United States, was ranked lowest on indicators of child well being as evidenced by perceived family support and relationship quality, risk taking behavior and general health (United Nations Children's Fund; UNICEF 2007). The report highlighted the ongoing need to investigate child and adolescent experiences of family life and how these experiences are related to risk taking and health related behavior. Children raised in families characterised by frequent expressions of anger and hostility, and within families whose relationships are cold and unsupportive are more vulnerable to mental and physical health problems, including behavior problems and substance abuse (Repetti et al. 2002). In particular, parenting behavior characterised by low warmth and high hostility has been associated with elevated conduct problems and smoking behavior (e.g., Melby et al. 1993). Cigarette use is often initiated in adolescence and places a considerable burden on smokers as well as society because of the high rates of associated morbidity and mortality (Leistikow 2000). Conduct problems negatively impact the quality of life of both the individual and those around them (Moffitt 2005). Children 
whose antisocial behavior persists into adulthood are at increased risk of a variety of problems, including those in the domains of mental health, substance dependence, economic difficulty and involvement in crime (e.g., KimCohen et al. 2003; Moffitt et al. 2002). There is a clear need to understand the underlying aetiology of cigarette use and conduct problems to be able to inform effective intervention at an early age.

Parent-Child Relationship Quality, Conduct Problems and Smoking Behavior

A wealth of research has identified expressions of hostility on the one hand, and low warmth on the other, as dimensions of parenting associated with increased risk of poor socio-emotional and behavioral development, including conduct problems (e.g., Ge et al. 1996a, b). Low parental affection or nurturing in adolescence has also been associated with elevated risk for antisocial personality disorder in adulthood (Johnson et al. 2006). In addition to conduct problems, poor family functioning, particularly in the parenting domain, is related concurrently and longitudinally with adolescent smoking (Chassin et al. 2005a; Tucker et al. 2002; van den Bree et al. 2004). Drawing on a social-developmental perspective of parental influence on adolescent behavior that emphasises how a hostile and rejecting rearing environment promotes and reinforces antisocial behavior in children (Patterson et al. 1989), research has shown effects of parents' low nurturance and hostility on adolescent's smoking behavior over and above peer influence and parent tobacco use (Melby et al. 1993). Substance misuse among children living in the context of dysfunctional family relationships may compensate for deficits in social and emotional development and serve a self-medicating function in response to dysregulation in the context of conflicted family life (Repetti et al. 2002). The documented links between parent expression of hostility and low warmth with adolescent behavior problems leave open the question of whether genetic and/or environmental effects underlie the relationship. By adopting a twin-study design that facilitates the disentangling of genetic and environmental effects that underlie the association between a psychosocial risk factor and adolescent adjustment, we investigated the relationship between parent-child relations with conduct problems and cigarette use.

Genetic and Environmental Influences on Appraisals of Family Functioning and Adolescent Adjustment

Twin studies examining adolescent cigarette use have indicated genetic, shared (environmental influences that make siblings alike) and non-shared environmental effects (influences that make siblings dissimilar), e.g., Fowler et al. (2007), Rhee et al. (2003). Conduct problems appear to be influenced by genetic factors, although here there is evidence for a stronger role of shared environment (Rhee and Waldman 2002). Research findings also suggest that the ways in which parents and children appraise family life and their relationships with other family members are also influenced, at least in part, by genetic factors (McGue et al. 2005; Neiderhiser et al. 2004). In other words, evidence indicates that genes influence the way parents relate and respond to the adolescent by contributing to the adolescent's behavior (Neiderhiser et al. 1998).

Parent-child relations and adolescent behavior problems may be associated by an overlap in genetic effects (e.g., common genetic factors) or by an overlap in environmental factors. Detecting significant common genetic effects indicates that the genetic effects on a given risk factor covary with those influencing the behavior of interest; in this instance, the genetic influences on parents' level of warmth and hostility are correlated with genetic influences on adolescent adjustment. Behavior genetics research has found evidence that children's experiences in the family are influenced by genetic factors and by non-shared environmental factors unique to that child. However, such insights have traditionally been based on decomposing variance in a given phenotype without actually measuring the nature of the non-shared environmental experience (Caspi et al. 2004). As Caspi et al. (2004) cogently argue, it is necessary to assess experiences that vary among children within families and investigate whether differences between children raised in the same family can account for variability in behaviors of interest. Current research increasingly incorporates measured psychosocial experiences of family life.

Previous investigations assessing the association between conflict and negativity in the parent-child relationship and antisocial behavior found evidence that these traits were influenced by genetic and environmental factors (Burt et al. 2003; Neiderhiser et al. 1998; Pike et al. 1996a). Longitudinal research has shown that, while children's genetically influenced externalizing behavior affects later levels of parent-child conflict, there is also evidence of environmentally mediated effects of parent-child conflict on externalising problems (Burt et al. 2005). Environmental mediation refers to covariation between the risk factor and behavior that is environmentally influenced. A recent longitudinal study of monozygotic twins further underscored the role of the non-shared environment by identifying maternal negative expressions of emotions towards a child as an environmental risk factor for 5-year old children's antisocial behaviour (Caspi et al. 2004). Evidence also has been found for environmental influence on the relationship between negative parenting and 
antisocial behaviour in older children and adolescents (O'Connor et al. 1998; Pike et al. 1996b). Collectively, research findings suggest a shared genetic liability to dysfunctional parent-child relationships and behaviour problems, together with the possible operation of nonshared environmentally mediated risk effects whereby parenting experiences not shared by siblings are associated with adolescent behaviour.

Despite significant progress made in considering links between poor parent-child relations, conduct problems and substance use, many studies have based findings on single-sex samples (e.g., Melby et al. 1993). Given adolescent girls' theorised proclivity for interpersonal connectedness and the risk this can pose for their mental health in the context of dysfunctional family functioning (Davies and Lindsay 2004), gender differences in the pattern of relations between parenting and adjustment are plausible. For instance, girls may be more likely to attempt to cope with negative parent-child relations by smoking cigarettes or by acting out their distress (behavior problems). On the other hand, given that maleness is a risk factor for conduct problems in adolescence (Rhee and Waldman 2002), associations between problematic parent-child relations and conduct problems may be more evident for boys. The extent to which the pattern of genetic and environmental influences on these associations is moderated by gender remains a relatively unexplored domain on inquiry, with further research required.

\section{The Present Study}

While previous research has documented genetic and environmental contributions to the association between parent negativity and behavior problems in adolescence, no research has examined the association between parenting and cigarette use with an adolescent sample using a genetically sensitive design. Indeed, a renewed emphasis on relations between family process and adolescent risk behavior is timely in light of research identifying (1) genetic influence on the initiation of cigarette use in adolescence and continued use (e.g., Fowler et al. 2007) and (2) links between poor parent-child relations and children's smoking behavior (e.g., Chassin et al. 2005a, b). Finally, while some research has examined the role of genetic and environmental influences underlying the relationship between parenting and adolescent behavior, few studies have examined the effects of using separate assessments of maternal and adolescent perceptions of parent behavior, despite previous research identifying differences in genetic and environmental effects as a function of the reporter of family functioning (e.g., Neiderhiser et al. 1998).
The present study is the first to examine genetic and environmental influences on the relationship between mother and adolescent appraisals of parent-to-child hostility and low warmth with both conduct problems and smoking cigarettes. Conduct problems and cigarette smoking were considered separately because cigarette smoking is increasingly conceptualised as distinct from other problem behaviors because of its addictive potential (Chassin et al. 2005b). While families with high levels of conflict and hostility often have low levels of acceptance and warmth, there is evidence that inadequate levels of emotional nurturance are independently associated with adjustment problems in children (Repetti et al. 2002). Therefore, levels of maternal warmth and hostility were also considered separately.

\section{Hypotheses}

We hypothesised that maternal hostility and low warmth would be positively associated with increased conduct problems and cigarette use. Based on previous research examining associations between parental negativity and conflict with antisocial behaviour (e.g., Neiderhiser et al. 1998; Caspi et al. 2004), we hypothesised that the association between mother-child relations with conduct problems and cigarette use would be primarily influenced by genetic factors and by non-shared environment effects. Finally, given the limited work conducted that examines effects separately by gender, we were particularly interested to explore the presence of gender differences in the pattern of relationships between mother-child relationships and adolescent adjustment and the potential for differential genetic and environmental influences therein.

\section{Method}

Sample

The data for the present analyses derived from the fourth wave (2004) of data collection of the longitudinal Cardiff Study of All Wales and North West of England Twins (CaStANET; van den Bree et al. 2007). The CaStANET register is a population-based twin register, including twins born between 1976 and 1991 in the Cardiff area of South Wales and between 1980 and 1991 for the rest of Wales and the North West of England. Questionnaires assessing various aspects of family functioning, parent health and twin psychological adjustment were mailed to families on the CaStANET twin register with twins aged 11-19 years. Questionnaire packs included instructions for completing the measures and separate stamped addressed envelopes for 
each family member to return their questionnaires. Parents and twins were asked to complete their questionnaires independently and a contact number for concerns or queries was provided. Non-responding families who might have changed address were traced through General Practitioners (Physicians). Of 1,755 families contacted, at least one family member from 1,214 families returned questionnaires, representing a response rate of $69 \%$. Family members who returned questionnaires received a gift voucher as a token of appreciation for their participation. Zygosity was assigned using a twin similarity questionnaire completed by the parents, which has been shown to be over 90\% accurate in distinguishing monozygotic (MZ) from dizygotic (DZ) twins (Nichols and Bilbro 1966). The CaStANET study received approval from the Multi Centre Research Ethics Committee for Wales, UK. Demographic statistics indicated that the sample was representative of British families living in the UK region of England and Wales with regard to family constitution, ethnicity, employment and economic factors (Social Trends 2004).

The present analyses are based on a sub-sample of 601 mothers and adolescent twin pairs living in single-parent or two-parent families, where twins were selected to be aged $12-17$ years old $($ mean $=15.28$ years; $S D=1.88)$. The final sample of twins comprised 231 monozygotic twins (100 male, 131 female pairs) and 370 dizygotic twins (72 male, 99 female, 199 opposite sex pairs). Both twins lived at home with their parent(s): the majority of twins lived with their biological mother and father $(71 \%)$ or mother only $(21 \%)$ while a smaller proportion lived with their biological mother and stepfather $(6.8 \%)$ or partner $(2.0 \%)$. Of the 1,214 families who replied, $6.2 \%$ were excluded from the present study because an adult other than the biological mother of the twins completed a questionnaire ( $4.5 \%$ fathers, $1 \%$ other, e.g., foster parent, grandparent). Families were also excluded from the present study if the twins were over 18 -years old ( $22 \%$ of responding families) or if the family composition was such that information was not provided about a resident mother-child relationship (23.8\% of the sample were living with their father only or 'other', e.g., foster family, one or both twins living away from home). Mothers with incomplete data on study variables reported lower levels of mother warmth and higher levels of mother hostility compared to those who formed the study sample (mother warmth, complete: mean $=$ 11.03, $\mathrm{SE}=0.20 ; \quad$ incomplete: mean $=12.26, \quad \mathrm{SE}=$ $0.54 ; t=2.14, \quad p<.05 ;$ mother hostility, complete: mean $=12.23, \mathrm{SE}=0.15$; incomplete: mean $=13.76$, $\mathrm{SE}=0.41 ; t=3.51, p<.01)$. Comparing families where the mother or a reporter other than the mother completed the parent questionnaire indicated that the parent/guardian and the adolescent reported lower levels of mother warmth (parent report, $t=3.43, p<.01$ and adolescent report, $t=2.96, p<.01)$. No other differences were found across study variables.

\section{Measures}

\section{Adolescent and Mother Reports of Mother Warmth and Hostility}

Adolescent and mother reports of mothers' warmth and hostility were measured using the Iowa Youth and Families Project parent warmth (four items) and hostility (five items) subscales (IYFP; Melby et al. 1993). Adolescents reported how often in the past month when they spent time talking or doing things together, the parent expressed warmth (example item, "Let you know she really cares about you") or hostility (example item, "Shout at you because she is upset with you"). Response options ranged from, 1 "Never" to 7 "Always". Items were coded to reflect low levels of expressed warmth and high levels of hostility. Adolescent reports demonstrated good internal consistency in the present sample (mother-child warmth, $\alpha=.91$ and hostility, $\alpha=.88$ ). Mothers answered equivalent questions and their reports demonstrated good internal consistency in the present sample (mother-child warmth, $\alpha=.79$ and hostility, $\alpha=.77$ ).

\section{Adolescent Conduct Problems}

Mother and adolescent reported conduct problems were assessed using the conduct problems subscale of the Strengths and Difficulties Questionnaire (Goodman 1997). Items included, "Often lies or cheats" "Steals from home, school or elsewhere", "Often fights with other young people or bullies them", "Often has temper tantrums or hot tempers" and "Generally obedient, usually does what adults request" (recoded to reflect disobedient behavior). Each item was rated 0 "Doesn't apply", 1 "Applies somewhat" or 2 "Certainly applies". Respondents reported on behavior over the last 6 months. Mother and adolescents' self reports of conduct problems demonstrated acceptable internal consistency in the present sample in ( $\alpha=.74$ and .70, for mothers and self report, respectively). Mother and adolescent reports were combined to give an overall index of conduct problems $(\alpha=.80)$.

\section{Adolescent Cigarette Use}

Smoking behavior was assessed as quantity of cigarettes smoked in the past month using a self-report question from the Add Health questionnaire (Resnick et al. 1997): 
"During the past month, on average, how many cigarettes did you smoke each day?" Response options ranged from 0 "Never had a cigarette in my life" to 7 "more than 30". A self-report measure of cigarette use was used because adolescents are likely to be the most reliable reporters of their own substance use behaviour. Previous research has indicated that adolescent self reports of cigarette smoking are valid (Wills and Cleary 1997) while the adolescent self report question was positively associated with mothers' reports of whether their child smoked or not $(r=.66$, $p<.01)$.

\section{Statistical Analysis}

Twin studies are based on the observation that monozygotic (MZ) twins share all of their genetic material while dizygotic (DZ) twins share approximately half of their genetic material in common. If a trait is genetically influenced, greater similarity is expected between MZ twins relative to DZ twins. A trait can be analysed to assess the proportion of variance attributable to additive genetic effects $\left(\mathrm{a}^{2}\right)$, shared environmental effects that make twins more similar $\left(\mathrm{c}^{2}\right)$ and non-shared environmental effects that make twins less similar $\left(\mathrm{e}^{2}\right)$. In the present study, twin analysis proceeded in two stages.

\section{Bivariate Genetic Modeling}

In studying whether associations between adolescent adjustment and relations with the mother are attributable to common genetic or environmental factors, one twin's adjustment scores can be correlated with the other twin's information on mother-child relations and vise versa. If these cross-twin cross-trait correlations are approximately twice as high in MZ than in DZ twins, this suggests that genetic influences play a role in the co-occurrence of the traits, while DZ cross-twin cross-trait correlations greater than half those of the MZ twins suggest shared environmental factors play a role (Neale and Cardon 1992).

In bivariate analyses, we examined the extent to which genetic, shared and non-shared environmental factors accounted for covariation in the association between mother-child relations, conduct problems and smoking behaviour. Using structural equation modelling, a bivariate correlated factors model was fit to the data (see Fig. 1). This is equivalent to a bivariate Cholesky decomposition model but makes no assumptions about the direction of causation (Loehlin 1996). Figure 1 presents the example of the relationship between mother-child hostility and conduct problems, assessing what proportion of the covariation between these two variables is attributable to genetic and

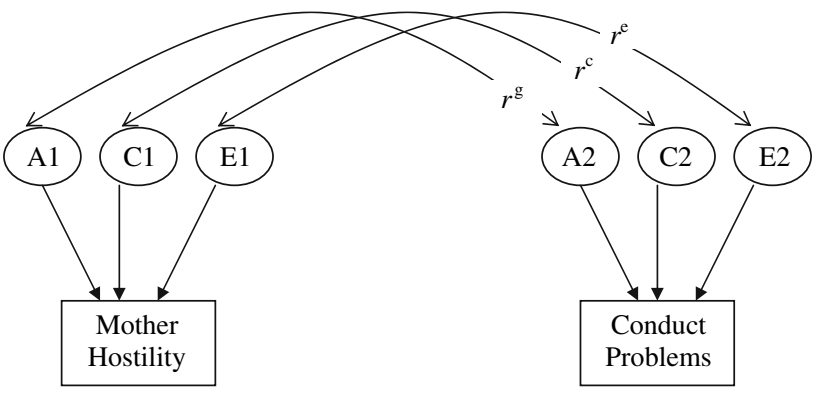

Fig. 1 A correlated factors model. Note: A conceptual correlated factors model of the relationship between mother-child hostility and adolescent conduct problems. Additive genetic, shared environment and unique environment variance contributions to mother hostility and adolescent conduct problems are indicated by A1, C1, E1 and A2, $\mathrm{C} 2, \mathrm{E} 2$, respectively. $r^{\mathrm{g}}, r^{\mathrm{c}}, r^{\mathrm{e}}$ represent the correlations between genetic, shared environment and non-shared environment contributions to mother hostility and conduct problems. For clarity of presentation, the figure depicts only one member of a twin pair

environmental influences. The bivariate correlated factors model gives estimates of the latent genetic $\left(r_{\mathrm{g}}\right)$, shared $\left(r_{\mathrm{c}}\right)$ and non-shared environmental $\left(r_{\mathrm{e}}\right)$ factors influencing the two traits (Neale and Cardon 1992). These correlations are independent of the size of the estimates of genetic and environmental influences on each of the variables of interest.

For all analyses, the entry of Twin 1 (first born) and Twin 2 (second born) data was randomised. To assess the significance of individual parameters, the goodness-of-fit of a model including estimates of the genetic, shared and non-shared environmental covariation was compared to a model in which each of these estimates was dropped. For clarity of presentation, results are presented for full bivariate models, including genetic and environmental estimates for each trait and the covariation between traits $\left(r^{\mathrm{g}}, r^{\mathrm{c}}, r^{\mathrm{e}}\right)$. The significance of these parameters was evaluated using $95 \%$ confidence intervals (CIs), where lower limits of 0 indicated non-significance. The software package Mx (Neale 1997) was used for structural equation modelling based on analysis of raw data with maximum likelihood estimation. For each model, the means for sex and age for adolescent twins were included as covariates.

\section{Regression Analyses of Monozygotic Twin Differences}

The MZ twin difference method is considered a strong, unambiguous test of non-shared environmental influences on a trait. Resemblance between MZ twins is attributable to shared genes and shared environmental factors, while they differ only with respect to non-shared environmental influences. Therefore, this method rules out genetic bias introduced by (1) a genetically transmitted liability accounting for correlations in mothers' parenting and 
adolescent behaviour and (2) genetically influenced differences between adolescents evoking differences in parenting (Caspi et al. 2004).

A regression approach was used where the difference score in parent-child relationship quality in monozygotic twins predicted a phenotypic difference score for conduct problems and cigarette use. The derived regression coefficient provides an estimate of the extent to which the relationship between two traits is due to non-shared environmental factors, independent of genetic and shared environmental factors. This can be represented as $Y_{1}-$ $Y_{2}=b_{0}+b_{1}\left(X_{1}-X_{2}\right)+\varepsilon$ (Purcell and Koenen 2005). For example, assessing non-shared environmental influences on the relationship between mother-child hostility and conduct problems can be represented as: conduct problems twin $1-$ conduct problems twin $2=b_{0}+b_{1}$ (mother hostility twin $1-$ mother hostility twin 2 ) $+\varepsilon$. Given that twins in each pair were randomly assigned as Twin 1 or Twin 2, difference scores were calculated by subtracting Twin 2's score on each measure of parent-child relations, conduct problems and cigarette use from Twin 1 's score.

\section{Results}

Table 1 contains the means, standard deviations, and correlations for all study variables. Preliminary analyses were conducted to examine the rate of cigarette use and to compare group level differences in study variables as a function of gender. About $14.6 \%$ of adolescents had smoked at least one cigarette in the past month. T-test comparisons across study variables were conducted using the survey commands in STATA 9.0 (StataCorp 2005), appropriate for use with twin data when there is nonindependence of observations. Comparisons for gender and zygosity indicated that males had higher levels of conduct problems compared to females (higher for males; mean $=4.45, \quad \mathrm{SE}=.02 ; \quad$ females $=4.33, \quad \mathrm{SE}=.02$, $t=4.36, p<.01)$. No other differences were found. Examining associations between twin age and each of the study variables indicated that age was positively correlated with cigarette use $(r=.25, p<.001)$ and negatively associated with conduct problems $(r=-.15, p<.001)$. Where significant associations were found between parentchild relations and age, these were small in magnitude (adolescent rated mother hostility, $r=.06, p<.05$ and warmth, $r=.10, p<.01$; mother rated low warmth, $r=.08, p<.01)$. Based on these results, age was included as a covariate in all twin analyses.

The correlations among study variables were positive and significant $(p<.01$; see Table 1). Adolescents' appraisals of mother hostility and low warmth were positively associated with conduct problems and with cigarette use. Mothers' reports of hostility and low warmth were also associated with conduct problems and cigarette use. A moderate positive association was found between cigarette use and conduct problems.

\section{Bivariate Genetic Analysis}

Based on inspection of the twin correlations and the crosstwin cross-trait correlations (see Table 2), which provide an initial indication of genetic and environmental influence, further analysis was conducted for the following relationships: (1) adolescents' reports of mother-child hostility with conduct problems and (2) cigarette use; (3) mothers' reported hostility and conduct problems; (4) adolescents' reports of mother-child low warmth with conduct problems and (5) cigarette use and (6) mothers' reports of low warmth and conduct problems. The results of bivariate genetic model fitting including standardized parameter estimates for genetic and environmental influence on each variable, and the association between variables, are presented in Table 3. Preliminary univariate model fitting indicated that the magnitude of genetic and environmental influences differed by gender for mother

Table 1 Phenotypic associations between parent-child relations, conduct problems and cigarette use

\begin{tabular}{|c|c|c|c|c|c|c|c|}
\hline & Variable & 1 & 2 & 3 & 4 & 5 & 6 \\
\hline 1. & Mother hostility (adolescent report) & - & & & & & \\
\hline 2. & Mother hostility (mother report) & $.48^{* *}$ & - & & & & \\
\hline 3. & Mother low warmth (adolescent report) & $.51 * *$ & $.27 * *$ & - & & & \\
\hline 4. & Mother low warmth (mother report) & $.27 * *$ & $.33 * *$ & $.43 * *$ & - & & \\
\hline 5. & Conduct problems & $.41 * *$ & $.52 * *$ & $.25^{* *}$ & $.23 * *$ & - & \\
\hline \multirow[t]{3}{*}{6.} & Cigarette use & $.22 * *$ & $.16^{* *}$ & $.21 * *$ & $.14 * *$ & $.32 * *$ & - \\
\hline & Mean & 11.67 & 12.23 & 13.62 & 11.03 & 4.39 & 1.69 \\
\hline & Standard deviation & 5.02 & 4.07 & 7.09 & 5.04 & 0.45 & 1.10 \\
\hline
\end{tabular}

Note: $\mathrm{N}=1,202 ; * * p<.01$ 
Table 2 Twin correlations and cross twin-cross trait correlations by zygosity for associations between parent-child relations and adolescent adjustment

\begin{tabular}{|c|c|c|c|c|c|c|}
\hline \multirow[t]{3}{*}{ Variables } & \multicolumn{2}{|c|}{ Twin correlations } & \multicolumn{4}{|c|}{ Cross-twin cross-trait correlations } \\
\hline & \multirow{2}{*}{$\begin{array}{l}\text { Monozygotic } \\
\text { twins } \\
(\mathrm{N}=462)\end{array}$} & \multirow{2}{*}{$\begin{array}{l}\text { Dizygotic } \\
\text { twins } \\
(\mathrm{N}=740)\end{array}$} & \multicolumn{2}{|c|}{ Monozygotic twins } & \multicolumn{2}{|l|}{ Dizygotic twins } \\
\hline & & & Twin $1-$ Twin 2 & Twin 2-Twin 1 & Twin 1-Twin 2 & Twin 2-Twin 1 \\
\hline Mother hostility (AR) \& cigarette use & $.25^{* *}$ & $.20 * *$ & $.24 * *$ & $.14^{*}$ & .09 & $.14^{* *}$ \\
\hline Mother hostility (AR) \& conduct problems & $.38 * *$ & $.42 * *$ & $.24 * *$ & $.29 * *$ & $.20 * *$ & $.12 *$ \\
\hline Mother hostility (MR) \& cigarette use & $.15^{* *}$ & $.17 * *$ & .12 & .11 & .05 & .10 \\
\hline Mother hostility (MR) \& conduct problems & $.56 * *$ & $.49 * *$ & $.45 * *$ & $.45 * *$ & $.20 * *$ & $.19 * *$ \\
\hline Mother warmth (AR) \& cigarette use & $.22 * *$ & $.19 * *$ & $.23 * *$ & $.16^{*}$ & $.13^{*}$ & .10 \\
\hline Mother warmth (AR) \& conduct problems & $.22 * *$ & $.27 * *$ & $.19 * *$ & $.21 * *$ & $.12 *$ & $.12 *$ \\
\hline Mother warmth (MR) \& cigarette use & $.18 * *$ & $.12 * *$ & $.15^{*}$ & $.16^{*}$ & $.15^{* *}$ & .08 \\
\hline Mother warmth (MR) \& conduct problems & $.26 * *$ & $.22 * *$ & $.20 * *$ & $.24 * *$ & $.11 *$ & .09 \\
\hline
\end{tabular}

Note: AR: adolescent report; MR: mother report; $* p<.05 ; * * p<.01$

reports of low warmth and for conduct problems (results available from the corresponding author). In bivariate model fitting with these study variables, the magnitude of genetic and environmental influence was allowed to vary for males and females.

Examining genetic and environmental effects at the individual trait level revealed that mother hostility (mother rated and adolescent rated) showed a significant genetic influence for males and significant non-shared environment effects for males and females (see column 1; Table 3). Mothers' rated hostility also showed a significant shared environment influence for both males and females. Adolescents' reports of maternal low warmth showed evidence of shared and non-shared environmental influence (with genetic influence found when effects were not estimated separately for males and females), while mothers' reports of warmth showed environmental influence only. Significant genetic and non-shared environment effects were found for male conduct problems; significant shared and non-shared environment effects were found for females (see column 2; Table 3). Significant genetic and nonshared environment effects were found for cigarette use.

Evidence was found for a significant genetic influence on the association between mother-child hostility and conduct problems and cigarette use (see column 3; Table 3). For mothers and adolescents' appraisals of hostility, the relationship with conduct problems showed a genetic influence for males, but not females. In addition to significant genetic effects, non-shared environmental correlations were found between mothers and adolescents' appraisals of hostility with conduct problems and cigarette use $\left(r^{\mathrm{e}}=.16-.38\right)$. There was a significant shared environmental correlation between mothers' reported hostility and female conduct problems $\left(r^{\mathrm{e}}=.43\right)$. The strong but non-significant shared environment correlations should be interpreted in the context of the large confidence intervals around these estimates $(-1.0,1.0)$, which reflect the small effect size of shared environment for adolescents' reports of mother-child relations and adjustment. In other words, these correlations are not substantively meaningful.

Shared environmental influence was found for the relationship between adolescents' appraisals of low warmth and female conduct problems $\left(r^{\mathrm{c}}=.41\right)$ and for mothers' reported low warmth and conduct problems $\left(r^{\mathrm{c}}=.79\right.$ and $r^{\mathrm{c}}=.37$ for males and females, respectively). For males, a genetic correlation was found between mothers' reported low warmth and conduct problems $\left(r^{\mathrm{g}}=.58\right)$, while for females, a significant non-shared environmental correlation was found $\left(r^{\mathrm{e}}=.21\right)$. Genetic and environmental effects were not found for the relationship between adolescents' reports of mothers' low warmth and cigarette use. Given the relatively small phenotypic association identified between these variables, this finding is not too surprising.

\section{Analysis of Monozygotic Twin Differences}

The correlations among MZ twin differences for all of the study variables are presented in Table 4 (gender differences were non-significant for all MZ twin difference correlations). Twin differences in adolescents' reports of mothers' hostility were associated with differences in conduct problems and cigarette use. Twin differences in mothers' reports of hostility were associated with conduct problems. Twin differences in mothers' reports of low warmth were associated with differences in conduct problems.

Regression analyses were conducted in which each index of MZ differences in mother-child relations was entered as a predictor of twin differences in conduct 


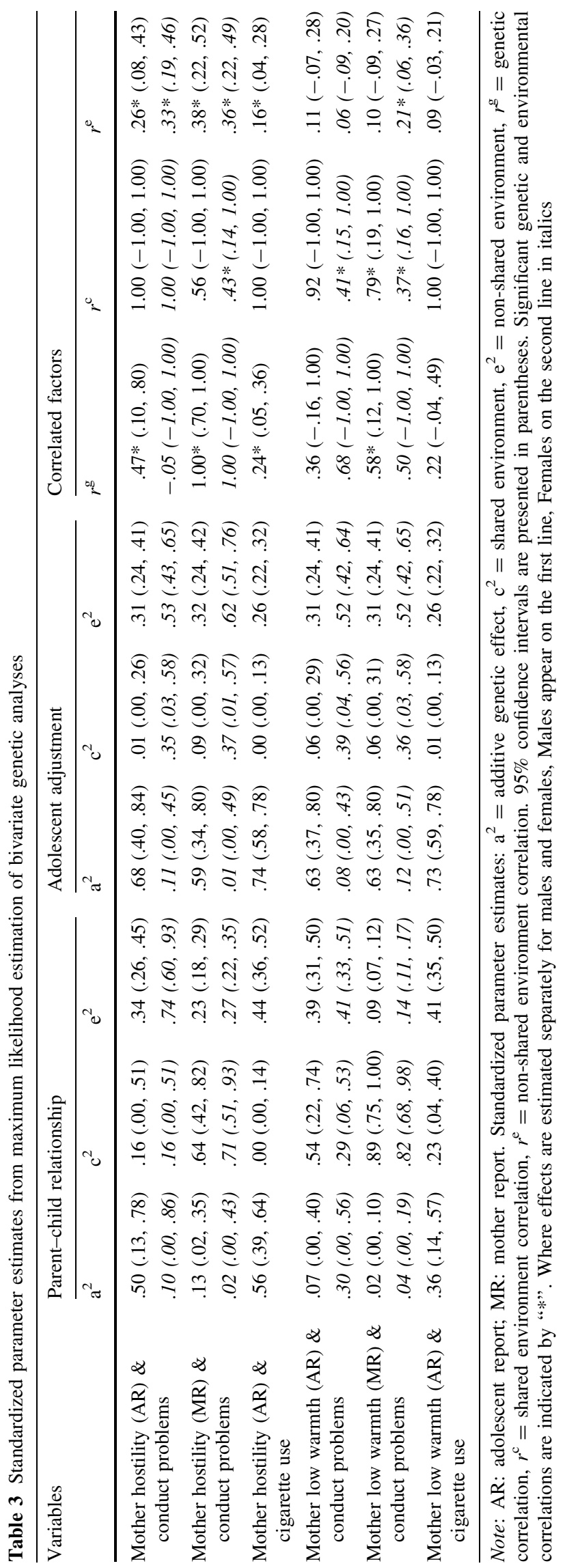


Table 4 Associations between monozygotic twin differences in mother-child relations, conduct problems and cigarette use

\begin{tabular}{|c|c|c|c|c|c|c|c|c|}
\hline & Variable & 1 & 2 & 3 & 4 & 5 & 6 & 7 \\
\hline 1. & Mother hostility (adolescent report) & - & & & & & & \\
\hline 2. & Mother hostility (mother report) & $.19 * *$ & - & & & & & \\
\hline 3. & Mother low warmth (adolescent report) & $.27 * *$ & .11 & - & & & & \\
\hline 4. & Mother low warmth (mother report) & .09 & $.45^{* *}$ & $.18 * *$ & - & & & \\
\hline 5. & Conduct problems & $.28 * *$ & $.37 * *$ & .03 & $.18 * *$ & - & & \\
\hline 6. & Cigarette use & $.17 *$ & .12 & .08 & .11 & $.29 * *$ & - & \\
\hline 7. & Conduct problems (1996) & .02 & $.17 *$ & .03 & .12 & $.18 *$ & .04 & - \\
\hline
\end{tabular}

Note: $\mathrm{N}=217 ; * p<.05 ; * * p<.01$

problems or cigarette use. When assessing the association between mother-child relations and conduct problems, a measure of MZ-twin differences in conduct problems at an earlier assessment (1996) was included as a covariate in an attempt to control for earlier non-genetic child effects eliciting negative parenting behavior (Moffitt 2005). This measure was the conduct problems subscale of the SDQ, rated by mothers $(\alpha=.64)$. In addition, to approximate the same statistical controls used in the bivariate genetic analysis, twin age and gender were included as covariates. The results of the regression analyses are presented in Table 5 .

Table 5 Regression analysis predicting monozygotic twin differences in conduct problems and cigarette use from twin differences in mother-child warmth and hostility

\begin{tabular}{lll}
\hline Regression model & $\beta$ & $t$ \\
\hline Conduct problems & & \\
Mother hostility (adolescent report) & & \\
Age & .09 & 1.42 \\
Gender & .06 & 0.96 \\
Mother hostility & $.29^{* *}$ & 4.44 \\
Conduct problems (Wave 1, 1996) & $.17^{* *}$ & 2.63 \\
Mother hostility (mother report) & & \\
Age & .05 & 0.79 \\
Gender & .01 & 0.13 \\
Mother hostility & $.35^{* *}$ & 5.32 \\
Conduct problems (Wave 1, 1996) & .11 & 1.73 \\
Mother warmth (mother report) & & \\
Age & .06 & 0.95 \\
Gender & .03 & 0.40 \\
Mother warmth & $.16^{*}$ & 2.28 \\
Conduct problems (Wave 1, 1996) & $.16^{*}$ & 2.31 \\
Cigarette use & & \\
Mother hostility (adolescent report) & & \\
Age & .03 & 0.46 \\
Gender & .06 & 0.87 \\
Mother hostility & $.19^{* *}$ & 2.96 \\
\hline
\end{tabular}

Note: $\mathrm{N}=217 ; * p<.05 ; * * p<.01$
The pattern of results showed that twin differences in mothers' and adolescents' reports of hostility predicted twin differences in conduct problems, after controlling for twin differences in earlier levels of conduct problems (Time $1 ; 1996)(\beta=.35, p<.01$ and $.29, p<.01$, for mother and adolescent report, respectively). Differences in adolescents' reports of mother hostility predicted differences in cigarette use $(\beta=.19, p<.01)$ while mothers' reports of low warmth predicted differences in conduct problems $(\beta=.16, p<.05)$.

\section{Discussion}

The present study investigated the extent to which genetic and environmental factors influenced relationships between maternal hostility and low warmth with adolescents' conduct problems and cigarette use. Based on the findings of two complementary approaches, support was found for the hypothesis that these relationships were influenced by both genetic and environmental effects. A notable finding was that differences between monozygotic twins in levels of mother warmth and hostility were related to increased conduct problems and cigarette use, providing strong support for environmental effects that are not shared by family members. These findings add to an emerging literature documenting genetic and environmental influences on links between well-defined indices of family functioning and adolescent adjustment.

Evidence was found for genetic and non-shared environmental influences on adolescents' appraisals of parentto-child warmth and hostility with no significant effects found for shared environment (with the exception of mothers' low warmth). For mothers' reports of parent behavior there was evidence of genetic, non-shared and shared environment effects for hostility and shared and non-shared environmental effects for low warmth. The difference in the relative role of shared and non-shared environment between child and parent reports may reflect parents' perceiving or portraying consistency in their 
behavior with each child, while children are more sensitive to and perceive greater differences between themselves, their siblings and their parents' treatment of them (Neiderhiser et al. 1998, 2004). However, when interpreting results of twin studies it should also be borne in mind that estimates of non-shared environmental influences include measurement error, which also tends to make twins less alike. Both cigarette use and conduct problems also showed significant genetic and non-shared environment effects. The magnitude of genetic effects is generally consistent with previous research examining these behaviors during adolescence, including the findings for conduct problems of a greater genetic estimate for males and the greater shared environment estimate for females (Rhee et al. 2003; Rhee and Waldman 2002).

The present study is among the first to consider gender differences in the genetic and environmental influences on indices of parent-child relationship quality. With the exception of mother reports of parent-child warmth, there was no evidence for sex differences in the genetic and environmental influences on indices of parent-child relations. There appeared to be a greater genetic effect for mothers' reports of warmth toward females and a greater shared environment effect for mothers' reports of warmth towards males. It is possible that mothers perceive greater continuity in their parenting towards males, while expressions of warmth towards females are influenced to a greater extent by the adolescent's genetically influenced characteristics and behavior. Overall, however, the results reveal similarity in the pattern of effects for males and females.

The pattern of associations between adolescents' appraisals of mothers hostility with cigarette use and conduct problems showed significant genetic influence. If hostile and aggressive behavior has a genetic basis, parents and children who share genes may engage in interpersonal behavior in the context of the family in ways that promote rather than attenuate the development of hostile behaviors (Repetti et al. 2002). This concurs with previous research (e.g., Pike et al. 1996a, b) and is consistent with the operation of gene-environment correlation, a process representing genetic control of exposure to environments (Purcell 2002). For example, genetically influenced adolescent characteristics (e.g., temperament) that affect parental behavior toward the child might also affect levels of conduct problems and cigarette use. This may occur because genetically influenced characteristics of the adolescents provoke (active gene-environment correlation) or evoke (evocative gene-environment correlation) negative behavior in the parent. Alternatively, adolescents' conduct problems and smoking behavior and the environment the parent provides are correlated because they have the same origins in the parents' genotype (passive gene-environment correlation; Rutter and Silberg 2002).
In addition to genetic effects, bivariate genetic analysis and regression analyses using monozygotic twin differences indicated that non-shared environmental influence were also important. The associations between mothers and adolescents' reports of hostility and conduct problems were significantly influenced by non-shared environmental factors. The consistency of effects for two different reporters of mothers' parenting behavior is promising and suggests environmental mediation reflects more than rater bias. Similarly, associations between mother-rated low warmth and conduct problems and between adolescent rated hostility and cigarette use were also partly accounted for by a significant non-shared environment effect. These findings suggest that negative parenting behavior towards different adolescents in the same family may account for individual differences in levels of conduct problems and substance use. Research has previously shown that differences in mothers' emotional attitudes toward their children are associated both concurrently and longitudinally with antisocial behavior (Caspi et al. 2004). These results extend this finding by documenting effects of non-shared environmental influence with an older age group of adolescent youth. Adolescents' adjustment problems may be a direct response to mothers' behavior or, alternatively, reflect increased affiliation with deviant peers that contributes to and/or compensates for poor parent-child relations (e.g., Brody and Forehand 1993). Investigating the processes underlying the genetic and environmental interplay between parent behavior, peer relations and adolescent adjustment is an important step for future research.

The results of this study provide a snapshot of genetic and environmental influences on the relationship between different family members' reports of the parent-child relationship and adolescent adjustment. However, as these analyses were cross-sectional and therefore did not test the direction of effects operating between parent-child relations and adolescent behaviors, this study was unable to differentiate between person effects on the environment and environmental effects on the individual (Rutter et al. 2001). Associations may be a function of environmentally determined behavioral differences between twins that lead to differential parental treatment and/or because differential parental treatment leads to differences in adolescent adjustment (Caspi et al. 2004). Cigarette use, for instance, may be part of a profile of genetically and environmentally influenced child behavior that leads parents to react in a less warm and more hostile manner. Adolescents with conduct problems or who smoke may incur parents' disapproval that reduces parental expressions of warmth and affection and increases family conflict. Although further research is required that uses a genetically sensitive, longitudinal design to disentangle the direction of effects between parenting, antisocial behavior and cigarette use 
over the course of adolescence, this study represents an initial exploration of such interplay.

The present study relied on a single index of cigarette use. The questions assessed in the present study formed part of a longer questionnaire assessing different dimensions of adolescents' lives, with efforts made to reduce item redundancy and respondent fatigue. There were practical reasons, therefore, for using a single item to assess frequency of cigarette use. However, this prevented assessing the internal consistency of the measure and may have misrepresented the pattern of smoking behavior in this sample of adolescents. However, the prevalence of smoking behaviour in this study was comparable to rates reported in large studies of substance use among young people in the UK (e.g., Hibell et al. 2004). Furthermore, a strong and significant correlation was found with mothers' reports of smoking by their children. Detailed assessment of smoking behavior including distinguishing between experimentation and more regular use of cigarettes, together with the context in which smoking occurred (e.g., alone or with friends) could be potentially informative for discerning links between family functioning and adolescent psychological adjustment. Although the item on smoking frequency has been used in national surveys of adolescent substance use (Johnston et al. 2005), given the complex cognitive, behavioral and physiological factors likely to characterise adolescent smoking, there have been calls to integrate pharmacological effects into models of psychosocial influence (Chassin et al. 2005a, b).

Finally, we did not examine links between father-child relations and adolescent behavior problems. The study from which these data derive did not collect information systematically from fathers; one parent from each family was asked to complete a questionnaire. Therefore, there were insufficient respondents to include father information as part of the twin analysis. Existing evidence suggests similarities in the pattern of genetic and environmental contributions to the association between mother- and fatheradolescent conflict and antisocial behavior (e.g., Neiderhiser et al. 1998). A related issue is the finding that in families where a respondent other than the mother completed the parent questionnaire, both the parent/guardian (usually the father) and the adolescent rated mothers lower in warmth. There are likely to be different reasons for these lower ratings, including that the adolescent completed the parent scale based on their relationship with a stepparent or other female guardian. Examining the pattern of associations across different family types and including father reports of parent-child relations to explore gender-based differences is an important area for future research.

In conclusion, these findings add to an emergent literature that uses a twin design to identify the effects of measured psychosocial influences on behavior problems by disentangling the contribution of genetic and environmental factors (Burt et al. 2003, 2005; Caspi et al. 2004; Pike et al. 1996a, b). Using two different methodological approaches, findings suggested that the relationships between mothers' expressions of warmth and hostility and adolescents' conduct problems and cigarette use were primarily accounted for by genetic factors and non-shared environmental influence. The results indicate that in addition to genetic contributions, differences in parental expressions of warmth and hostility may account for differences in levels of conduct problems and cigarette use for adolescents from the same family. With this in mind, the targeting of resources at the level of policy and intervention should retain an emphasis on the support of families while paying increased attention to the individual needs of adolescents living within families and their subjective experience of the parent-child relationship. Given the evidence for both genetic and environmental effects, interventions that aim to assist parents to adopt behaviors that enable children and adolescents to develop self-regulation and adaptive behavior in response to stress may be most effective (Repetti et al. 2002). More generally, the findings underscore the value of considering different family members' perspectives on family life and links therein to adolescent risk behavior. Evidence for the operation of environmental effects highlights opportunities for targeting interventions aimed at reducing cigarette use and conduct problems while further research that examines the temporal nature of these relationships is also required.

Acknowledgements We would like to thank the families who participated in the Cardiff Study of All Wales and North West of England Twins for their time and contribution to this project. Ivan Nikolov from the Biostatistics Bioinformatics Unit, Department of Psychological Medicine at Cardiff University provided database management and support. The development of this manuscript was supported by grants awarded by the Wellcome Trust (GR073063) to Marianne van den Bree, Anita Thapar, Michael C. Neale, Gordon Harold and Jane Scourfield and by the European Research Advisory Board awarded to Marianne van den Bree, Anita Thapar and Jane Scourfield. Support was also provided by an NIH grant (DA-018673) awarded to Michael C. Neale. Thanks are extended to Terrie E. Moffitt, Ph.D. and Michael J. Owen, MD for helpful comments provided.

Open Access This article is distributed under the terms of the Creative Commons Attribution Noncommercial License which permits any noncommercial use, distribution, and reproduction in any medium, provided the original author(s) and source are credited.

\section{References}

Brody, G. H., \& Forehand, R. (1993). Prospective associations among family form, family processes, and adolescents' alcohol and drug use. Behavior Research \& Therapy, 31, 587-593.

Burt, S. A., Krueger, R. F., McGue, M., \& Iacono, W. G. (2003). Parent-child conflict and the comorbidity among childhood 
externalizing disorders. Archives of General Psychiatry, 60, 505-513.

Burt, S. A., McGue, M., Krueger, R. F., \& Iacono, W. G. (2005). How are parent-child conflict and childhood externalizing symptoms related over time? Results from a genetically informative crosslagged study. Development \& Psychopathology, 17, 145-165.

Caspi, A., Moffitt, T. E., Morgan, J., Rutter. M., Taylor, A., Arseneault, L., Tully, L., Jacobs, C., Kim-Cohen, J., \& PoloTomas, M. (2004). Maternal expressed emotion predicts children's antisocial behavior problems: Using monozygotic-twin differences to identify environmental effects on behavioral development. Developmental Psychology, 40, 149-161.

Chassin, L., Presson, C. C., Rose, J., Sherman, S. J., Davis, M. J., \& Gonzalez, J. L. (2005a). Parenting style and smoking-specific parenting practices as predictors of adolescent smoking onset. Journal of Pediatric Psychology, 30, 333-344.

Chassin, L., Presson, \& Sherman, S. J. (2005b). Adolescent cigarette smoking: A commentary and issues for pediatric psychology. Journal of Pediatric Psychology, 30, 299-303.

Davies, P. T., \& Lindsay, L. L. (2004). Interparental conflict and adolescent adjustment: Why does gender moderate early adolescent vulnerability? Journal of Family Psychology, 8, $160-170$.

Fowler, T., Lifford, K., Shelton, K., Rice, F., Neale, M., Thapar, A., McBride, A., \& van den Bree, M. B. M. (2007). Exploring the relationship between genetic influences on initiation and progression of substance use. Addiction, 101, 413-422.

Ge, X., Best, K., Conger, R. D., \& Simons, R. L. (1996a). Parenting behaviors and the occurrence and co-occurrence of adolescent depressive symptoms and conduct problems. Developmental Psychology, 32, 717-731.

Ge, X., Conger, R. D., Cadoret, R. J., Neiderhiser, J. M., Yates, W., Troughton, E., \& Stewart, M. A. (1996b). The developmental interface between nature and nurture: A mutual influence model of child antisocial behavior and parent behaviors. Developmental Psychology, 32, 574-589.

Goodman, R. (1997). The Strengths and Difficulties Questionnaire: A research note. Journal of Child Psychology and Psychiatry, 38, $581-586$.

Hibell, B., Andersson, B., Bjarnason, T., Ahlström, S., Balakireva, O., Kokkevi, A., et al. (2004). The ESPAD Report 2003. Alcohol and other drug use among students in 35 European countries. Stockholm, Sweden: The Swedish Council for Information on Alcohol and Other Drugs (CAN) and the Pompidou Group at the Council of Europe.

Johnson, J. G., Cohen, P., Chen, H., Kasen, S., \& Brook, J. S. (2006). Parenting behaviors associated with risk for offspring personality disorder during adulthood. Archives of General Psychiatry, 63, 579-587.

Johnston, L. D., O’Malley, P. M., Bachman, J. G., \& Schulenberg, J. E. (2005). Monitoring the Future national results on adolescent drug use: Overview of key findings, 2004 (NIH Publication No. 05-5726). Bethesda, MD: National Institute on Drug Abuse.

Kim-Cohen, J., Caspi, A., Moffitt, T. E., Harrington, H., Milne, B. J., \& Poulton, R. (2003). Prior juvenile diagnoses in adults with mental disorder: Developmental follow-back of a prospective-longitudinal cohort. Archives of General Psychiatry, 60, 709-717.

Leistikow, B. N. (2000). The human and financial costs of smoking. Clinics in Chest Medicine, 21, 189-197.

Loehlin, J. C. (1996). The Cholesky approach: A cautionary note. Behavior Genetics, 26, 5-69.

McGue, M., Elkins, I., Walden, B., \& Iacono, W. G. (2005). Perceptions of the parent adolescent relationship: A longitudinal investigation. Developmental Psychology, 41, 971-984.
Melby, J., Conger, R. D., Conger, K. J., \& Lorenz, F. O. (1993). Effects of parental behavior on tobacco use by young male adolescents. Journal of Marriage and the Family, 55, 439-454.

Moffitt, T. E. (2005). The new look of behavioral genetics in developmental psychopathology: Gene-environment interplay in antisocial behaviors. Psychological Bulletin, 131, 533-554.

Moffitt, T. E., Caspi, A., Harrington, H., \& Milne, B. J. (2002). Males on the life-course persistent and adolescent-limited antisocial pathways: Follow-up at age 26 years. Development and Psychopathology, 14, 179-207.

Neale, M. C. (1997). Mx: Statistical modeling (Computer software and manual) (4th ed.). Richmond: Medical College of Virginia, Department of Psychiatry.

Neale, M. C., \& Cardon, L. R. (1992). Methodology for genetic studies of twins and families. Dordrecht, Netherlands: Kluwer Academic Publishers.

Neiderhiser, J. M., Pike, A., Hetherington, E. M., \& Reiss, D. (1998). Adolescent perceptions as mediators of parenting: Genetic and environmental contributions. Developmental Psychology, 34, 1459-1469.

Neiderhiser, J. M., Reiss, D., Pedersen, N. L., Lichtenstein, P., Spotts, E. L., Hansson, K., Cederblad, M., \& Elthammer, O. (2004). Genetic and environmental influences on mothering of adolescents: A comparison of two samples. Developmental Psychology, 40, 335-351.

Nichols, R. C., \& Bilbro, W. C., Jr. (1966). The diagnoses of twin zygosity. Acta Genetics of Statistical Medicine, 16, 265-275.

O'Connor, T. G., Deater-Deckard, K., Fulker, D., Rutter, M., \& Plomin, R. (1998). Genotype environment correlations in late childhood and early adolescence: Antisocial behavioral problems and coercive parenting. Developmental Psychology, 34, 970-981.

Patterson, G. R., DeBaryshe, B. D., \& Ramsey, E. (1989). A developmental perspective on antisocial behavior. American Psychologist, 44, 329-335.

Pike, A., McGuire, Reiss, D., Hetherington, E. M., \& Plomin, R. (1996a). Family environment and adolescent depressive symptoms and antisocial behavior: A multivariate genetic analysis. Developmental Psychology, 32, 590-603.

Pike, A., Reiss, D., Hetherington, E. M., \& Plomin, R. (1996b). Using MZ differences in the search for non-shared environmental effects. Journal of Child Psychology \& Psychiatry, 37, 695-704.

Purcell, S. (2002). Variance components models for gene-environment interaction in twin analyses. Twin Research, 5, 554-571.

Purcell, S., \& Koenen, K. C. (2005). Environmental mediation and the twin design. Behavior Genetics, 35, 491-498.

Repetti, R. L., Taylor, S. E., \& Seeman, T. E. (2002). Risky families: Family social environments and the mental and physical health of offspring. Psychological Bulletin, 128, 330-366.

Resnick, M. D., Bearman, P. S., Blum, R. W., Bauman, K. E., Harris, K. M., Jones, J., Tabor, J., Beuhring, T., Sieving, R. E., Shew, M., Ireland, M., Bearinger, L. H., \& Udry, J. R. (1997). Protecting adolescents from harm. Findings from the National Longitudinal Study on Adolescent Health. JAMA, 278, 823-832.

Rhee, H. R., Hewitt, J. K., Young, S. E., Corley, R. P., Crowley, T. J., \& Stallings, M. C. (2003). Genetic and environmental influences on substance initiation, use, and problem use in adolescents. Archives of General Psychiatry, 60, 1256-1264.

Rhee, S. H., \& Waldman, I. R. (2002). Genetic and environmental influences on antisocial behavior: A meta-analyses of twin and adoption studies. Psychological Bulletin, 128, 490-529.

Rutter, M., Pickles, A., Murray, R., \& Eaves, L. (2001). Testing hypotheses on specific environmental effects on behavior. Psychological Bulletin, 127, 291-324. 
Rutter, M., \& Silberg, K. (2002). Gene-environment interplay in relation to emotional and behavioral disturbance. Annual Review of Psychology, 53, 463-490.

Social Trends (2004). London: Office of National Statistics.

Statacorp (2005). Stata statistical software: Release 9. College Station, TX: Statacorp LP.

Tucker, J. S., Ellickson, P. L., \& Klein, D. J. (2002). Five-year prospective study of risk factors for smoking in adolescence among early non-smokers and experimenters. Journal of Applied Social Psychology, 32, 1588-1603.

UNICEF (2007). Child poverty in perspective: An overview of child well-being in rich countries, Innocenti Report Card 7. Florence: UNICEF Innocenti Research Centre.

van den Bree, M., Rice, F., Fowler, T., Shelton, K., Lifford, K., Scourfield, J., Harold, G., \& Thapar, A. (2007). The Cardiff Study of All Wales and North West of England Twins (CaStANET): A longitudinal research programme of child and adolescent development. Twin Research on Human Genetics.

van den Bree, M. B. M., Whitmer, M. D., \& Pickworth, W. B. (2004). Predictors of smoking development in a population-based sample of adolescents: A prospective study. The Journal of Adolescent Health, 35, 172-181.

Wills, T. A., \& Cleary, S. D. (1997). The validity of self-reports of smoking: Analyses by race/ethnicity in a school sample of urban adolescents. American Journal of Public Health, 87, 56-61.

\section{Author Biographies}

Katherine H. Shelton (Ph.D., 2003, Cardiff University) is a Research and Teaching Fellow in the School of Psychology at Cardiff University. Her research interests focus on family influences on children's psychological development. In particular, she is interested in highlighting factors that promote resilience and ameliorate risk for children living in households marked by hostile interparental relations.

Gordon Harold (Ph.D., 1998, Cardiff University) is a Reader in the School of Psychology at Cardiff University. His research interests focus on the role of the family as a context for understanding children's normal and abnormal psychological development, the genetic origins of children's emotional and behavioral development, policy and practice applications of research relating to family influences on children's development, and the application of statistical modelling techniques to the analysis of longitudinal data.

Tom Fowler (Ph.D., 2003, Cardiff University) is a Specialist Trainee (SPT) in Public Health and an Honorary Research Fellow in Primary Care and General Practice at the University of Birmingham. His research interests relate to chronic fatigue, antisocial behaviour and substance use in adolescence. He has a specific interest in applying research findings to the public health setting, particularly those relating to adolescent behaviour.

Frances Rice (Ph.D., 2002, Cardiff University). Her research work focuses on the origins and outcomes of depression during childhood and adolescence. A number of her research papers examine the link between depression in parents and depression in children. She has a particular research interest in the co-action and inter-action of genetic and psychosocial factors in childhood psychopathology.

Michael Neale obtained his B.Sc. and Ph.D. in Psychology from the University of London in the 1980s, and is now Professor of Psychiatry and Human Genetics at the Virginia Institute for Psychiatric and Behavioral Genetics. He specializes in the development of statistical models and software for fitting them to genetically informative datasets.

Anita Thapar (MRCPsych., 1989, Royal College of Psychiatrists) qualified in medicine in 1985. In 1999, she was appointed as the first Professor in Child and Adolescent Psychiatry in Wales. Her research focuses on the interface of genetic epidemiology, psychosocial and clinical research in child and adolescent psychiatry.

Marianne van den Bree (Ph.D., 1994, Virginia Commonwealth University) is a Senior Lecturer in the Department of Psychological Medicine at Cardiff University. Her research interests relate to how genes and the environment influence psychological/psychiatric traits and particularly the complex pathways of these influences and their interactions over time, from childhood through adolescence to adulthood. She is also interested in mental health and social problems in homeless and prison populations. 